\title{
EFECTOS DE ALGUNOS FACTORES METEOROLÓGICOS SOBRE LA CONCENTRACIÓN DE ESPORAS DE HONGOS EN LA PLAZA SAN MARTÍN DE LIMA
}

\author{
EFFECTS OF SOME METEOROLOGICAL FACTORS ON THE \\ CONCENTRATION OF FUNGAL SPORES AT \\ PLAZA SAN MARTÍN, LIMA, PERÚ
}

\author{
Roberto Ramos ${ }^{1}$ y Víctor Meza ${ }^{1}$
}

\begin{abstract}
Resumen
Se realizó un estudio aeromicológico para determinar la influencia de algunos factores meteorológicos en la concentración de esporas fúngicas totales en la atmósfera de la Plaza San Martín de Lima. El muestreo se realizó utilizando el método volumétrico por impactación empleando un muestreador tipo Andersen con agar Sabouraud. Las concentraciones más altas de esporas se observaron en los meses de marzo y setiembre mientras que los niveles más bajos fueron detectados en los meses de julio y agosto. Los hongos predominantes que estuvieron presentes en todo el periodo de estudio fueron Cladosporium, Penicillium y Aspergillus, estos géneros abarcaron el $82 \%$ del total de esporas aisladas. La concentración de esporas totales mostró una correlación estadística significativa $(\mathrm{p}<0.05)$ positiva con la temperatura e índice $U V$; la humedad relativa presentó una correlación estadística significativa $(\mathrm{p}<0.05)$ negativa. La velocidad del viento presentó una correlación estadística positiva con un nivel de significancia de $\mathrm{p}<0.01$. Las fluctuaciones de las condiciones meteorológicas muestran una marcada influencia en la distribución de las esporas.
\end{abstract}

Palabras clave: aeromicología, bioaerosol, esporas, alergia, variables climáticas.

\begin{abstract}
An aeromicological study was carried out to establish the influence of meteorological factors on the concentration of total fungal spores in the atmosphere of the Plaza San Martín, Lima, Perú. Sampling was carried out using the volumetric impaction method using an Andersen sampler with Sabouraud agar. The highest concentrations of spores was observed in the months of March and September while the lowest levels were detected in the months of July and August. The predominant fungi that were present throughout the study period were Cladosporium, Penicillium and Aspergillus. These genera comprised $82 \%$ of the total isolated spores. The total spore concentration showed a significant statistical correlation $(\mathrm{p}<0.05)$ with temperature and UV index while relative humidity presented a negative significant statistical correlation $(\mathrm{p}<0.05)$. Wind speed presented a positive statistical correlation with a level of significance of $p<0.01$. Fluctuations of meteorological conditions showed a marked influence on the distribution of the spores.
\end{abstract}

Key words: aeromycology, bioaerosol, airborne spore, allergy, meteorological factors.

Introducción.

Las esporas de los hongos son los componentes más abundantes de los bioaerosoles, su concentración y composición se ve influenciada por factores biológicos y ambientales, siendo las variables meteorológicas (temperatura, humedad relativa, velocidad del viento) uno de los más importantes que influyen fuertemente en la dispersión de las esporas en la atmósfera (Stępalska \& Wolek, 2005; Abdel Hameed et al., 2012; Grinn-Gofroń \& Bosiacka, 2015). Los estudios aeromicológicos nos permiten conocer la concentración de las esporas fúngicas presentes en la atmósfera y dar una mejor comprensión de la relación entre sus concentraciones y las variables meteorológicas (Grinn-Gofroń \& Bosiacka, 2015).
El aire es un ambiente natural para las esporas de muchos hongos y a pesar de su reducido tamaño y una dispersión importante tienen un gran impacto en la salud humana y en otras áreas como la producción agrícola (Jędryczka, 2014). Los más frecuentes en el aire son Cladosporium, Penicillium, Aspergillus y Alternaria, sus concentraciones son altas independientemente de la latitud y altitud sobre el nivel del mar. Otras especies de hongos cuyas esporas están presentes en el aire incluyen a Fusarium, Sclerotinia y Ganoderma. El monitoreo aerobiológico es ampliamente usado en epidemiología de las enfermedades humanas (alergias) e infecciones de plantas (Jędryczka, 2014). 
Oliveira et al. (2009) mencionan que los hongos son típicamente más abundantes en verano y otoño. Varios factores climáticos como la humedad, temperatura, velocidad del viento, índice UV y presión atmosférica afectan la producción y dispersión de los hongos (Sabariego et al., 2000; Sakiyan \& Inceoglu, 2003; Kumari et al., 2011). Sin embargo, no existe información sobre el efecto de los factores meteorológicos sobre la dispersión de las esporas en la atmósfera de las localidades urbanas de la ciudad de Lima, metrópoli considerada como una de las más densamente pobladas en América Latina y con relativamente altos valores de polución (CarbajalArroyo et al., 2007).

La Plaza San Martín de Lima es una fuente natural de aerosoles, generados por las personas que circulan, las fuentes de agua, la vegetación, la persistente llovizna y las aves que las habitan. Sin embargo, no existen estudios sobre la concentración de microorganismos presentes en los aerosoles y sus probables consecuencias en la salud de los pobladores, así como también el efecto de las estaciones del año y los cambios atmosféricos en la estabilidad de los bioaerosoles.

El presente estudio evaluó la influencia de los factores meteorológicos sobre la concentración de los propágulos totales en la atmósfera de la Plaza San Martín de Lima.

Materiales y métodos.

Ubicación del área de estudio.

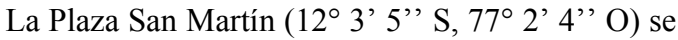
encuentra ubicada en la ciudad de Lima $\left(12^{\circ} 2\right.$ ' $45^{\prime \prime} \mathrm{S}$, $77^{\circ} 1$ ' 50" O), capital del Perú, a $101 \mathrm{~m}$ sobre el nivel del mar. Bordea el litoral, aproximadamente, en el km. 50 de la Panamericana Norte desde el distrito de Ancón, hasta el distrito de Pucusana, este último cercano al km 70 de la Panamericana Sur. Su clima es de tipo desértico subtropical, tiene una temperatura ambiental que oscila entre $12{ }^{\circ} \mathrm{C}$ en invierno, y $28{ }^{\circ} \mathrm{C}$ en verano, la humedad relativa varía entre 80 y $100 \%$ durante todo el año. Se eligieron 5 puntos de muestreo que tomaron en cuenta los factores generadores de aerosoles y que, en conjunto, describieron una cruz y abarcaron zonas dentro del perímetro delimitado por los Jirones Unión, Ocoña, Carabaya, y Nicolás de Piérola.

Muestreo del aire.

Los muestreos fueron realizados una vez por mes, de marzo a septiembre de 2014 entre las 10 y 11 horas por el método volumétrico por impactación utilizando un equipo de impacto en cascada tipo Andersen (Andersen, 1958). El flujo de aire aspirado fue de 28.3 1/min y el muestreo duró 5 minutos por vez y fue ejecutado a $1.5 \mathrm{~m}$ de altura. El aire se aspiró sobre placas Petri con agar Sabouraud en cada punto de muestreo para el aislamiento de hongos, procedimiento que fue realizado por duplicado de modo secuencial. Los recuentos totales de colonias cultivables se presentaron como unidades formadoras de colonias en el aire $\left(\mathrm{UFC} / \mathrm{m}^{3}\right)$. Para este cálculo, se utilizó un factor de corrección para el conteo de colonias, basado en la probabilidad de que más de un propágulo viable podría haber pasado a través del mismo agujero e impactado en el medio de cultivo. El cálculo se realizó de acuerdo a la fórmula de Feller (Andersen, 1958):

$$
\mathrm{UFC} / \mathrm{m}^{3}=\mathrm{N} x \ln [\mathrm{N} /(\mathrm{N}-\mathrm{P})]
$$

Donde $\mathrm{N}$ fue el número total de orificios de la placa, $\mathrm{P}$ es el número de colonias que crecieron en el medio. El resultado se dividió entre un factor de conversión de litros a $\mathrm{m}^{3}(\mathrm{~K}=0.1415)$.

Cultivo e identificación de hongos.

Para el recuento y posterior aislamiento de los hongos, las placas Petri con agar Sabouraud se incubaron durante 5 días a $25{ }^{\circ} \mathrm{C}$. Los hongos filamentosos fueron identificados por observación macroscópica y microscópica de las hifas y formas de reproducción asexual, empleando la técnica de la cinta adhesiva para su reconocimiento hasta género. Las colonias que presentaron dificultades en su identificación se cultivaron en cámara húmeda para su posterior identificación (Ellis, 1971, 1976; Ainsworth et al., 1995; Barnett \& Hunter, 1998).

Variables meteorológicas.

Los parámetros meteorológicos fueron proporcionados por el Servicio Nacional de Meteorología e Hidrología (SENAMHI) a través de su estación meteorológica ubicada en el Campo de Marte situada a $2 \mathrm{Km}$ de la unidad de monitoreo y por el Instituto Metropolitano Protransporte de Lima (PROTRANSPORTE). Se midieron variables como temperatura, humedad relativa, velocidad del viento e índice UV. Las variables meteorológicas medidas corresponden al momento en que se realizó el muestreo. Análisis estadístico de los datos.

La homogeneidad de varianzas entre las distribuciones mensuales de las esporas atmosféricas fue analizada por ANOVA de un solo factor. El análisis de las diferencias significativas entre la distribución mensual de las esporas totales $\left(\mathrm{UFC} / \mathrm{m}^{3}\right)$ fue seguido por la prueba de Tukey $(\mathrm{p}<0.05)$. Se realizó un análisis de correlación de Pearson entre las variables meteorológicas y la concentración de esporas totales $\left(\mathrm{UFC} / \mathrm{m}^{3}\right)$ para evaluar la posible asociación entre las variables meteorológicas y la concentración de esporas fúngicas totales. La temperatura, velocidad del viento, humedad relativa e índice UV fueron determinadas como variables independientes, mientras la concentración de esporas totales $\left(\mathrm{UFC} / \mathrm{m}^{3}\right)$ fue establecido como variable dependiente. El test de correlación de Pearson fue estudiado con niveles de significancia de 0.05 y 0.01 (bilateral) usando el software estadístico SPSS, versión 20.

\section{Resultados.}

Se identificaron 40 géneros de hongos filamentosos, de ellos, tres fueron aislados más frecuentemente: 
Tabla 1. Concentración promedio de esporas totales y variables climáticas en la Plaza San Martín de Lima.

\begin{tabular}{lccccccc}
\hline & Marzo & Abril & Mayo & Junio & Julio & Agosto & Setiembre \\
\hline $\mathrm{UFC} \mathrm{m}^{3}$ & 468 & 355 & 315 & 275 & 117 & 225 & 368 \\
Temperatura $\left({ }^{\circ} \mathrm{C}\right)$ & 25.7 & 21.9 & 21.2 & 17.4 & 15.9 & 15.8 & 16.3 \\
Humedad relativa $(\%)$ & 54 & 73 & 74 & 80 & 90 & 89 & 83 \\
Velocidad del viento $(\mathrm{m} / \mathrm{s})$ & 1.8 & 1.9 & 1.6 & 1.2 & 0.6 & 1.1 & 1.6 \\
Índice UV & 11.1 & 8.0 & 3.0 & 2.0 & 2.0 & 3.0 & 3.0 \\
\hline
\end{tabular}

Cladosporium (51\%), Penicillium (25\%) y Aspergillus (6\%). También se identificaron Alternaria (1.7\%), Fusarium (0.8\%), Acremonium (0.7\%), Curvularia (0.7\%), Cylindrocarpon (0.5\%), Sclerotinia (0.5\%), Ulocladium $(0.5 \%)$, \%), Bipolaris $(0.1 \%)$, Mucor $(0.1 \%)$, Rhizopus $(0.1 \%)$ entre otros. Con respecto a las levaduras se aisló en mayor porcentaje Rhodotorula $(2.3 \%)$.

Se encontraron diferencias significativas $(\mathrm{p}<0.05)$ entre la concentración de esporas totales y los meses del año. Los meses de marzo y septiembre muestran diferencias significativas respecto a los otros meses (Figura 1). La concentración promedio de esporas totales fue más alta en marzo $\left(468 \mathrm{UFC} / \mathrm{m}^{3}\right)$ y setiembre (367 UFC/m $\mathrm{m}^{3}$ ) mientras que los valores más bajos fueron detectados en julio $\left(117 \mathrm{UFC} / \mathrm{m}^{3}\right)$ y agosto $(225$ $\left.\mathrm{UFC} / \mathrm{m}^{3}\right)$. La velocidad del viento fue más alta en marzo $(1.8 \mathrm{~m} / \mathrm{s})$ y abril $(1.9 \mathrm{~m} / \mathrm{s})$ y los valores más bajos fueron detectados en julio $(0.6 \mathrm{~m} / \mathrm{s})$ y agosto $(1.1 \mathrm{~m} / \mathrm{s})$. La temperatura del aire fue más alta en marzo $\left(25.7^{\circ} \mathrm{C}\right)$ y abril $\left(21.9^{\circ} \mathrm{C}\right)$, mientras que los valores más bajos fueron detectados en julio $\left(15.9^{\circ} \mathrm{C}\right) \mathrm{y}$ agosto $\left(15.8^{\circ} \mathrm{C}\right)$. Además, la humedad relativa fue más alta en julio $(90$ $\%)$ y agosto ( $89 \%$ ), mientras los valores más bajos se alcanzaron en marzo (54\%) y abril $(73 \%)$. El índice UV fue más alta en marzo (11.1) y abril (8.0), mientras los valores más bajos fueron obtenidos en junio (2.0) y

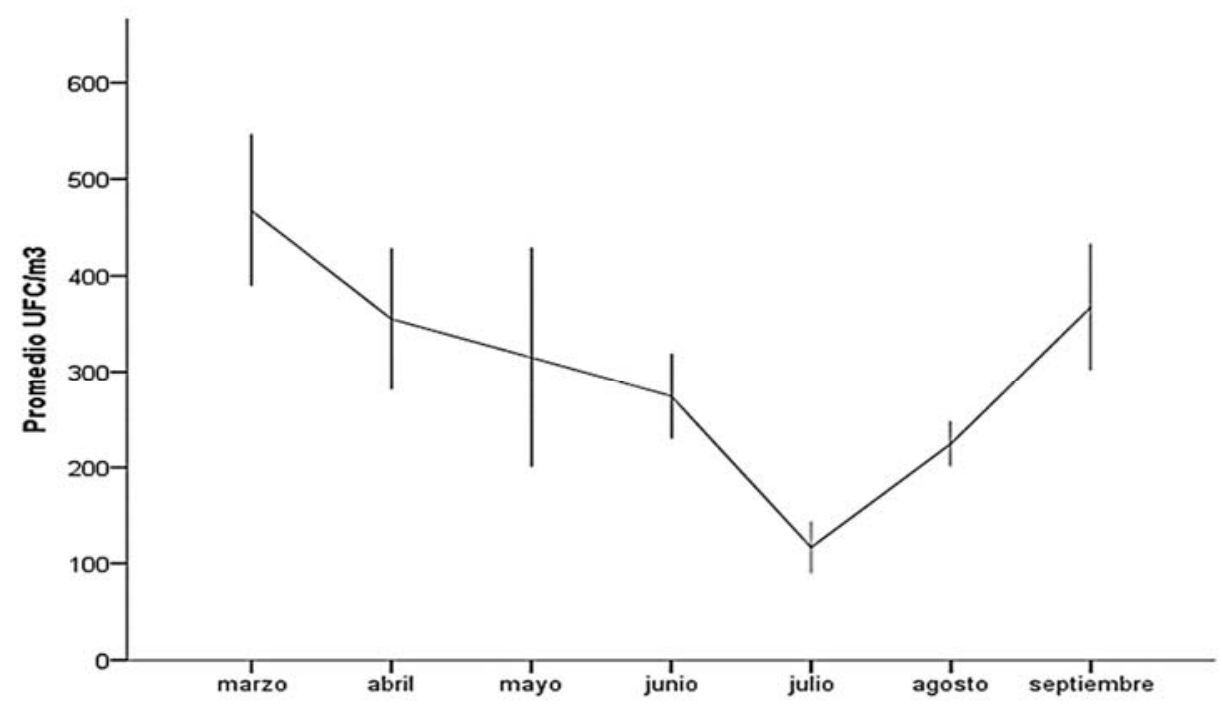

Figura 1. Concentraciones promedio de esporas fúngicas totales en la atmósfera de la Plaza San Martín. La barra indica la desviación estándar. julio (2.0). Un resumen de las variables meteorológicas y la carga microbiana se muestra en la Tabla 1.

Respecto a la influencia de las variables climáticas sobre la concentración de esporas totales $\left(\mathrm{UFC} / \mathrm{m}^{3}\right)$ (Figura 2), se ha encontrado que existe una correlación significativa positiva con la temperatura $(\mathrm{R}=0.768$, $\mathrm{p}<0.05)$, velocidad del viento $(\mathrm{R}=0.924, \mathrm{p}<0.01)$ e índice UV $(\mathrm{R}=0.759, \mathrm{p}<0.05)$ (Tabla 2). Con relación a la otra variable climática, la humedad relativa presentó una correlación significativa negativa $(\mathrm{R}=-0.854$, $\mathrm{p}<0.05$ ) (Tabla 2).

\section{Discusión.}

Este estudio aeromicológico es el primero en realizarse en Lima (Perú), no existe publicaciones sobre la concentración de esporas en el aire exterior en nuestro país.

La diversidad de hongos en el aire es poco conocida, su estudio en el aire exterior e interior no solamente es importante como patógenos y alérgenos para los humanos, animales y plantas, sino que también es relevante para la ecología porque pueden, potencialmente, influir en la química y física atmosférica, la biosfera y el clima al actuar como núcleos de condensación y cristalización de gotas de agua y hielo en nubes y niebla (Fröhlich-Nowoisky et al., 2009). El crecimiento de los hongos presenta una marcada periodicidad estacional relacionadas con las 
CONCENTRACIÓN DE ESPORAS DE HONGOS POR FACTORES METEOROLÓGICOS EN LIMA Julio - Diciembre 2017

Tabla 2. Análisis de correlación de Pearson.

\begin{tabular}{lcccc}
\hline & $\begin{array}{c}\text { Velocidad del viento } \\
(\mathrm{m} / \mathrm{s})\end{array}$ & $\begin{array}{c}\text { Temperatura } \\
\left({ }^{\circ} \mathrm{C}\right)\end{array}$ & $\begin{array}{c}\text { Humedad relativa } \\
(\% \mathrm{HR})\end{array}$ & Índice UV \\
\cline { 2 - 5 } Concentración de esporas & $\mathrm{R}=0.924^{* *}$ & $\mathrm{R}=0.768^{*}$ & $\mathrm{R}=-0,854^{*}$ & $\mathrm{R}=0.759^{*}$ \\
$(\mathrm{p}=0.003)$ & $(\mathrm{p}=0.044)$ & $(\mathrm{p}=0.014)$ & $(\mathrm{p}=0.048)$ \\
\hline $\left.\mathrm{UFC}^{3}\right)$ & & & &
\end{tabular}

condiciones meteorológicas. Diferentes autores han encontrado una variación en la concentración de esporas respecto a las condiciones meteorológicas. (Quintero et al., 2010; Almaguer et al., 2013; Almaguer et al., 2015; Guarín et al., 2015). Así, Lin \& Li (2000) investigaron las relaciones entre la concentración de esporas y las condiciones meteorológicas. Ellos encontraron que las concentraciones de esporas fueron altamente dependientes de las condiciones climáticas, con concentraciones máximas cuando la temperatura fue de $25-30{ }^{\circ} \mathrm{C}$, la humedad relativa fue de $60-70 \%$, la velocidad del viento fue menor que $1 \mathrm{~m} / \mathrm{s}$. En consecuencia, una variación en la temperatura, humedad relativa, precipitación, velocidad del viento, presión atmosférica, horas de luz solar, etc. afectarán la concentración de esporas en el aire (Ho et al., 2005; Fang et al., 2005; Kumar et al., 2011).

En nuestro estudio se encontró que la velocidad del viento mostró una fuerte correlación significativa positiva $(\mathrm{R}=0.924, \mathrm{p}<0.01)$ con la concentración de las esporas totales (Figura 2a). Esta situación se compara con lo observado por Calderón et al. (1997), ellos encontraron una correlación positiva entre la velocidad del viento y la concentración de esporas, observaron que se obtiene una concentración más alta generalmente cuando la velocidad del viento y la temperatura fueron suficientemente altas como para romper la cadena conidial; esta observación coincide con nuestros resultados, como se muestra en la tabla 1 , en la que se advierte que la máxima concentración de esporas fue en marzo lo que coincide con una velocidad del viento y temperatura más alta. Lin \& Li (2000) afirmaron que la influencia de la velocidad del viento como factor de dilución o sobrevivencia de los bioaerosoles ha sido largamente demostrada en modelos de dispersión y estudios ambientales. Mallaiah \& Rao (1982) mencionan que el viento es el responsable del desprendimiento y de la dispersión de las esporas en la atmósfera, cualquier movimiento del aire puede liberar algunas esporas, pero cuando la velocidad del viento excede $0.5 \mathrm{~m} / \mathrm{s}$ el número de esporas liberadas se incrementa rápidamente $\mathrm{y}$ este efecto puede continuar hasta una velocidad del viento de $3-5 \mathrm{~m} / \mathrm{s}$.

En nuestra investigación también se ha encontrado que existe una fuerte correlación significativa positiva entre la concentración de esporas totales con la temperatura $(\mathrm{R}=0.768, \mathrm{p}=0.044) \quad$ (Figura 2b), al respecto, Kurkela (1997), en Finlandia, menciona que, aunque la temperatura está correlacionada significativamente con el número de esporas en el aire su efecto fue más importante en la producción de esporas más que para la liberación o dispersión de las mismas. Quintero et al. (2010) analizando qué variables meteorológicas están asociadas con la concentración de esporas encontraron una fuerte correlación con la temperatura, humedad relativa, velocidad del viento entre otras variables climáticas. El índice UV mostró una correlación significativa positiva, con un nivel de significancia de $p<0.05$. La mayor concentración de esporas coincide con los mayores niveles de índice UV (Figuras 2d). Esto indica que la radiación UV estimula la esporulación y conidiación, en efecto, Rodríguez-Romero et al. (2010) observaron que la luz ultravioleta $(350-370 \mathrm{~nm})$ y la luz azul (450 nm) actúan como un represor de la reproducción sexual favoreciendo su esporulación. También se ha reportado que la luz UV de longitud de onda larga estimula la esporulación de varios hongos (Leach, 1971; Tuite, 1969). Dahlberg \& Etten (1982) han demostrado también que longitudes de onda entre 300 a $380 \mathrm{~nm}$, cercana a la región UV induce más esporulación que aquellas en el rango UV (200-300 $\mathrm{nm})$. Esta última región podría ser letal o mutagénica. Finalmente, Bayram et al. (2010) estudiando el efecto de la luz sobre el desarrollo de los hongos encontró que la reproducción sexual y asexual son dependientes de la luz; en la oscuridad se produce más estructuras sexuales mientras que la exposición a la luz verde claro predominan la producción de conidiosporas.

Con relación a la humedad relativa se encontró que existe una correlación significativa negativa $(\mathrm{R}=-0.854$, $\mathrm{p}=0.014$ ) (Figura 2c), al respecto Reponen et al. (1996) concluyeron que las esporas de los hongos son ligeramente higroscópicos, asimismo, Pasanen et al. (1991), estudiando la influencia de la humedad del aire y la velocidad del viento en la liberación de esporas en la atmósfera de Finlandia, encontraron que la concentración de esporas es más alta cuando la humedad del aire es más baja obteniendo un crecimiento mínimo cuando la humedad relativa es mayor al 70 \%. Zoberi (1961) encontró que una disminución en la humedad del aire aceleró la liberación de esporas. Nevalainen et al. (1998) estudiando hogares en Finlandia con problemas de hongos encontraron que el conteo de esporas fue muy bajo a pesar de que el crecimiento del hongo es claramente visible, esto se explica por el hecho de que los hongos no esporulan ni liberan sus esporas continuamente, sino que la liberación depende de la humedad del aire y la temperatura. Finalmente, Pasanen et al. (1991) concluyó que el tamaño de las esporas está determinado por la humedad del aire, cuanta más humedad existe en la atmósfera el tamaño 


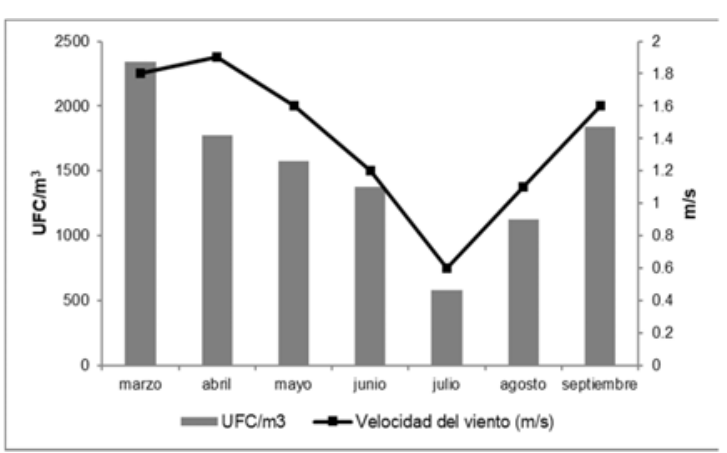

a) Relación entre la velocidad del viento y la concentración de esporas totales

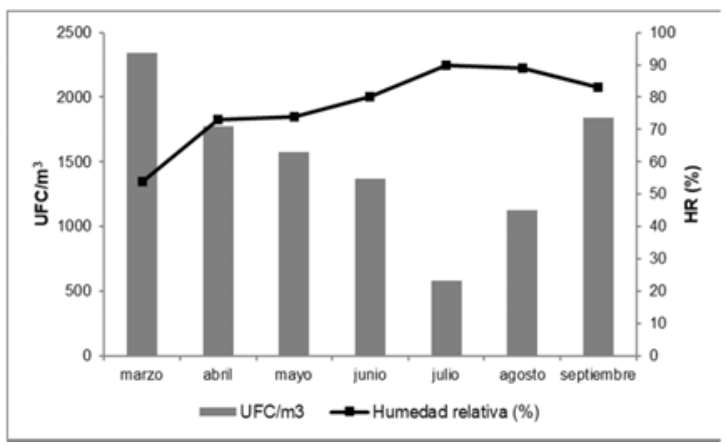

c) Relación entre la humedad relativa y la concentración de esporas totales

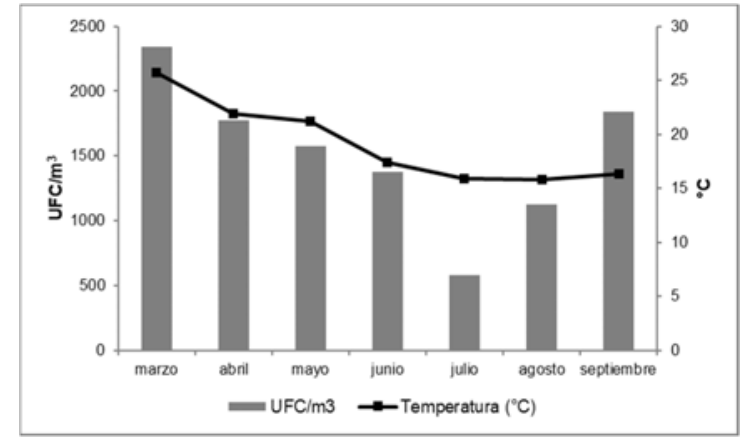

b) Relación entre temperatura y la concentración de esporas totales

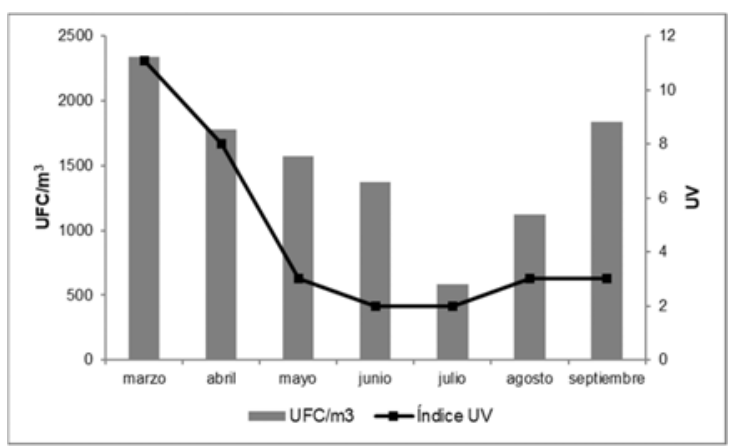

d) Relación entre el índice UV y la concentración de esporas totales

Figura 2. Relación entre los factores meteorológicos y la concentración de esporas fúngicas totales.

aerodinámico de la espora es más grande lo cual afecta su diseminación.

Con relación a la concentración total de esporas en el aire, se halló que la máxima concentración de esporas fue en la estación de verano (marzo) y luego en la primavera (setiembre). Principalmente, en el verano se observó un evidente incremento en la concentración de esporas junto con un incremento en la velocidad del viento y la temperatura del aire. Estos resultados concuerdan con Kasprzyk (2008), quienes obtuvieron la concentración más alta de esporas en el verano o a inicios del otoño; Hasnain (1993), encontró una fuerte tendencia hacia un incremento en la concentración de esporas durante los meses más calurosos y una gradual disminución durante el invierno; Stępalska \& Wolek (2005) encontraron que la máxima concentración de esporas fue en el verano. Quintero et al. (2010) afirman que, en regiones templadas, el verano y el otoño son las estaciones con la más alta concentración de esporas en la atmósfera debido a la presencia de altas cantidades de sustrato orgánico, importante para organismos descomponedores como los hongos. En contraste,
Quintero et al. (2010) también han reportado que en pocas regiones tropicales como en San Juan (Puerto Rico), la concentración de esporas durante el año presenta un determinado patrón estacional que está más relacionado a los patrones de precipitación. Estos resultados indican que la velocidad del viento y la temperatura del aire son los parámetros meteorológicos más importantes que afectan la concentración de esporas en el aire (Adams, 1964; McCartney, 1994; Kasprzyk, 2008).

Durante el período de muestreo, las esporas predominantes en la atmósfera de la Plaza San Martín de Lima fueron Cladosporium, Penicillium y Aspergillus. Estos hongos son conocidos como los más severos en la inducción de alergias (Lin \& Li, 2000; Green et al. 2006; Quintero et al., 2010). En el Perú, no existe estudios sobre la inducción de alergias por Cladosporium, Penicillium y Aspergillus por lo que se recomienda realizar estudios sobre estos hongos alergénicos. 


\section{Conclusiones.}

La presencia de esporas en la atmósfera de la Plaza San Martín de Lima, está fuertemente influenciada por las condiciones microclimáticas. La concentración de esporas totales mostró una correlación significativa positiva con la temperatura del aire, velocidad del viento e índice UV; la humedad relativa presentó una correlación significativa negativa. También, la más alta concentración de esporas ocurrió en el mes de marzo. Finalmente, Cladosporium, Penicillium y Aspergillus estuvieron presentes en todo el período de estudio, como los hongos aeroalergénicos más comunes.

\section{Agradecimientos.}

Municipalidad Metropolitana de Lima, Instituto Metropolitano Protransporte de Lima (PROTRANSPORTE); Servicio Nacional de Meteorología e Hidrología (SENAMHI) y un agradecimiento especial al Laboratorio de Ecología de Artrópodos (LEA), UNALM.

\section{Literatura citada.}

Abdel Hameed A. A., Khoder M. I., Ibrahim Y. H., Saeed Y., Osman M. E. \& Ghanem S. 2012. Study on some factors affecting survivability of airborne fungi. Science of the Total Environment, 414, 696-700.

Adams KF. 1964. Year to year variation in the fungus spore content of the atmosphere. Acta Allergol. 19:11-50.

Ainsworth G. C., Bisby G. R., Hawksworth D. L., Kirk P. M., Sutton B. C. and Pegler D. N. 1995. Dictionary of the Fungi. 8th ed. Wallingford: CAB International.

Almaguer M., Aira M-J., Rodríguez-Rajo F-J., \& Rojas T-I. 2013. Study of airborne fungus spores by viable and nonviable methods in Havana, Cuba. Grana, 52(4), 289-298.

Almaguer M., Aira M. J., Rodríguez-Rajo F. J., FernandezGonzalez M. \& Rojas-Flores T. I. 2015. Thirty-four identifiable airborne fungal spores in Havana, Cuba. Annals of Agricultural and Environmental Medicine, 22(2), 215-220.

Andersen A. 1958. New sampler for the collection, sizing and enumeration of viable airborne particles. Journal of Bacteriology, 76(5), 471-484.

Barnett H. \& Hunter B. 1998. Illustrated Genera of Imperfect Fungi. 4th ed. APS Press, St. Paul, Minnesota.

Bayram O., Biesemann C., Krappmann S., Galland P. \& Braus G.H. 2008. More than a repair enzyme: Aspergillus nidulans photolyase-like CryA is a regulator of sexual development. Mol. Biol. Cell. 19, 3254-3262.

Calderón C., Lacey J., McCartney A. \& Rosas I. 1997. Influence of urban climate upon distribution of airborne Deuteromycete spore concentrations in Mexico City. Int J Biometeorol. 40, 71-80.

Carbajal-Arroyo L., Barraza-Villarreal A., Durand-Pardo R., Moreno-Masías H., Espinoza-Laín R., Chiarella-Ortigosa P. \& Romieu I. 2007. Impact of traffic flow on the asthma prevalence among school children in Lima, Peru. Journal of Asthma; 44 (3): 197-202.

Dahlberg KR. \& Etten J. 1982. Physiology and biochemistry of fungal sporulation. Annu Rev Phytopathol. 20(1):281301.
Ellis MB. 1971. Dematiaceous hyphomycetes. Commonwealth Mycological Institute, Kew.

Ellis MB. 1976. More dematiaceous hyphomycetes. Commonwealth Mycological Institute, Kew.

Fang Z., Ouyang Z., Hu L., Wang X., Zheng H. \& Lin X. 2005. Culturable airborne fungi in outdoor environments in Beijing, China. Science of the Total Environment, 350(1-3), 47-58.

Fröhlich-Nowoisky J., Pickersgill D., Després V. R. \& Pöschl U. 2009. High diversity of fungi in air particulate matter. Proceedings of the National Academy of Sciences of the United States of America, 106(31), 12814-12819.

Green BJ., Tovey ER., Sercombe JK., Blachere FM., Beezhold DH. \& Schmechel D. 2006. Airborne fungal fragments and allergenicity. Med Mycol. 44:245-55.

Grinn-Gofroń A. \& Bosiacka B. 2015. Effects of meteorological factors on the composition of selected fungal spores in the air. Aerobiologia, 31(1), 63-72.

Guarín F. A., Alberto M., Abril Q., Alvarez A. \& Fonnegra R. 2015. Atmospheric pollen and spore content in the urban area of the city of Medellin, Colombia. Hoehnea, 42(1), 9-19.

Hasnain SM. 1993. Influence of meteorological factors on the air spora. Grana. 32:184-188.

Ho H. M., Rao C. Y., Hsu H. H., Chiu Y. H., Liu C. M. \& Chao H. J. 2005. Characteristics and determinants of ambient fungal spores in Hualien, Taiwan. Atmospheric Environment, 39(32), 5839-5850.

Horner W., Helbling A., Salvaggio J. \& Lehrer S. 1995. Fungal allergens. Clinical Microbiol Rev. 2:161-179.

Jędryczka M. 2014. Aeromycology: studies of fungi in aeroplankton. Folia Biologica et Oecologica, 10(1), 1826.

Kasprzyk I. 2008. Aeromycology - Main research fields of interest during the last 25 years. Ann Agric Environ Med. 15:1-7.

Kumar P., Mahor P., Goel A. K., Kamboj D. V. and Kumar O. 2011. Aero-microbiological study on distribution pattern of bacteria and fungi during weekdays at two different locations in urban atmosphere of Gwalior, Central India. Scientific Research and Essays, 6(25), $5435-5441$.

Kumari S., Gond D. K., Samuel C. O. \& Abassi P. 2011. A comparative study of aeromycospora in different localities of Gorakhpur, U. P. Indian J. Sci. Res., 2(4), 5155.

Kurkela T. 1997. The number of Cladosporium conidia in the air in different weather conditions. Grana. 36:54-61.

Leach C. M. 1971. A practical guide to the effects of visible and ultraviolet light on fungi. pp. 609- 664. In Booth C. (ed.) Methods in Microbiology. Vol 4. Academic Press, New York, 795p.

Lin W. \& Li C. 2000. Associations of fungal aerosols, air pollutants, and meteorological factors. Aerosol Sci Tech. 32:359-68.

Mallaiah K. \& Rao AS. 1982. Aerial dissemination of uredinospores of groundnut rust. Trans Br Mycol Soc. 78:21-28.

McCartney A. 1994. Dispersal of spores and pollen from crops. Grana. 33:76-80.

Nevalainen A., Partanen P., Jääskeläinen E., Hyvärinen A., Koskinen O., Meklin T., Vahteristo M., Koivisto J. \& Husman T. 1998. Prevalence of moisture problems in Finnish houses, Indoor Air, 4(Suppl.), 45-49. 
Oliveira M., Ribeiro H., Delgado J. L., \& Abreu I. 2009. Aeromycological profile of indoor and outdoor environments. Journal of Environmental Monitoring: JEM, 11(7), 1360-1367.

Pasanen A. L., Pasanen P., Jantunen M. J. \& Kalliokoski P. 1991. Significance of air humidity and air velocity for fungal spore release into the air. Atmospheric Environment Part A, General Topics, 25(2), 459-462.

Quintero E., Rivera-Mariani F. \& Bolaños-Rosero B. 2010. Analysis of environmental factors and their effects on fungal spores in the atmosphere of a tropical urban area (San Juan, Puerto Rico). Aerobiologia, 26(2), 113-124.

Reponen T., Willeke K., Ulevicius V., Reponen A. \& Grinshpun S. A. 1996. Effect of relative humidity on the aerodynamic diameter and respiratory deposition of fungal spores. Atmospheric Environment, 30(23), 39673974.

Rodríguez-Romero J., Hedtke M., Kastner C., Müller S. \& Fischer R. 2010. Fungi, hidden in soil or up in the air:
Light makes a difference. Annu Rev Microbiol. 64:585610.

Sabariego S., Díaz de la Guardia C. \& Alba F. 2000. The effect of meteorological factors on the daily variation of airborne fungal spores in Granada (southern Spain). International Journal of Biometeorology, 44(1), 1-5.

Sakiyan N., Inceoglu O. 2003. Atmospheric concentrations of Cladosporium Link and Alternaria Nées spores in Ankara and the effects of meteorological factors. Turk J Bot. 27, $77-81$.

Stępalska D. \& Wołek J. 2005. Variation in fungal spore concentrations of selected taxa associated to weather conditions in Cracow, Poland, in 1997. Aerobiologia 21:43-52.

Tuite J. 1969. Plant pathological methods, fungi and bacteria. pp.141 - 175. Burgess Publ., Minneapolis, Minn., 239p.

Zoberi MH. Take-off of mold spores in relation to wind speed and humidity. Ann Botany. 1961; 25: 53-64.

\footnotetext{
${ }^{1}$ Departamento de Biología. Universidad Agraria La Molina, Facultad de Ciencias. Av. La Molina S/N, La Molina. Lima Perú. roberto@1amolina.edu.pe
} 